\title{
Heavy flavour photoproduction at HERA
}

\author{
Verena Schönberg * \\ Physikalisches Institut der Universität Bonn \\ Nußallee 12, 53115 Bonn, Germany \\ E-mail: vschoenb@physik.uni-bonn.de
}

\begin{abstract}
Charm and beauty photoproduction has been studied in ep collisions at HERA with the ZEUS and $\mathrm{H} 1$ detectors. Heavy quarks were identified using different experimental techniques. Charm was identified via the reconstruction of $\mathrm{D}^{*}$ mesons, while beauty was tagged via its semi-leptonic decay into leptons or using lifetime tagging techniques. Differential cross sections were measured and compared to leading order plus parton shower (LO+PS) Monte Carlo and next-to-leading order (NLO) QCD predictions.
\end{abstract}

European Physical Society Europhysics Conference on High Energy Physics, EPS-HEP 2009, July 16 - 222009

Krakow, Poland

*on behalf of the H1 and ZEUS collaborations 


\section{Introduction}

Heavy flavour production in $e^{ \pm} p$ collisions at HERA provides a good testing ground of perturbative Quantum Chromodynamics (QCD) as the high quark masses provide a hard scale. The analyses presented in this talk focus on the photoproduction regime, i.e. the photon virtuality, $Q^{2}$, is required to fulfill $Q^{2} \approx 0 \mathrm{GeV}^{2}$. The measurements are compared to next-to-leading order (NLO) QCD predictions, for the calculation of which most of the analyses made use of the FMNR programme [1]. The programme is based on the Fixed Flavour Number Scheme (FFNS), in which the heavy flavours are generated dynamically in the matrix elements and no initial beauty or charm is assumed in the proton. This is valid for $\mu^{2} \approx O\left(m_{q}^{2}\right)$, with $q$ being the beauty or charm quark. Another scheme in use is the General Mass Variable Flavour Number Scheme (GMVFNS [2]), which also treats the heavy quarks as massive at $\mu^{2} \approx O\left(m_{q}^{2}\right)$, but as massless and part of the proton at $\mu^{2} \gg m_{q}^{2}$.

Different techniques can be used and combined to tag the quarks, such as meson tagging (e.g. $D^{*}$ tagging), lepton tagging using the decay spectra and lifetime tagging exploiting the long lifetime of the heavy hadrons. Various tracking improvements in both experiments allow for a more precise determination of impact parameters and secondary vertices when using data collected in the HERA II (2003-2007) data taking period. The most recent results using these methods are presented here.

\section{2. $\mathbf{D}^{*}$ production}

In this analysis done by the $\mathrm{H} 1$ collaboration [3] the charm quark is tagged by a $D^{*}$ meson decaying in the golden decay channel $D^{* \pm} \rightarrow D^{0} \pi_{\text {slow }}^{ \pm} \rightarrow K^{\mp} \pi^{ \pm} \pi_{\text {slow }}^{ \pm}$. A dataset corresponding to a luminosity of $93 \mathrm{pb}^{-1}$ with events in the kinematic range $Q^{2}<2 \mathrm{GeV}^{2}, 100<W<$ $285 \mathrm{GeV}, p_{t}\left(D^{*}\right)>1.8 \mathrm{GeV}$ and $\left|\eta\left(D^{*}\right)\right|<1.5$ was selected. The number of $D^{*}$ mesons was determined from the $M(K \pi \pi)-M(K \pi)$ distribution, which is displayed in Fig. 1. Figure 2 shows the differential cross sections as a function of $p_{t}\left(D^{*}\right)$ and $\eta\left(D^{*}\right)$. The measurements were compared with two NLO QCD predictions, one using the Fixed Flavour Number Scheme (FFNS) within the FMNR programme and the other one using the General Mass Variable Flavour Number Scheme (GMVFNS). The data were found to be in reasonable agreement with both predictions; GMVFNS has a softer $p_{t}\left(D^{*}\right)$ spectrum and a slightly different shape in $\eta\left(D^{*}\right)$ compared to FFNS and the measurement. Furthermore, double-

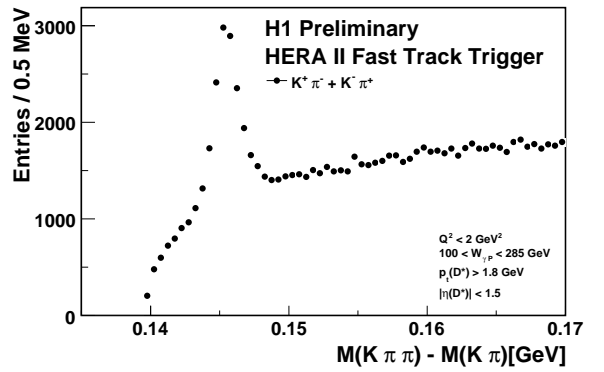

Figure 1: $M(K \pi \pi)-M(K \pi)$ distribution. differential cross sections as a function of $p_{t}\left(D^{*}\right)$ and $\eta\left(D^{*}\right)$ were measured. Again good agreement between the measurements and both NLO predictions was observed in general; however, the theoretical uncertainties are very large and both predictions fail to describe the data at high $p_{t}\left(D^{*}\right)$ and high $\eta\left(D^{*}\right)$ [3]. 

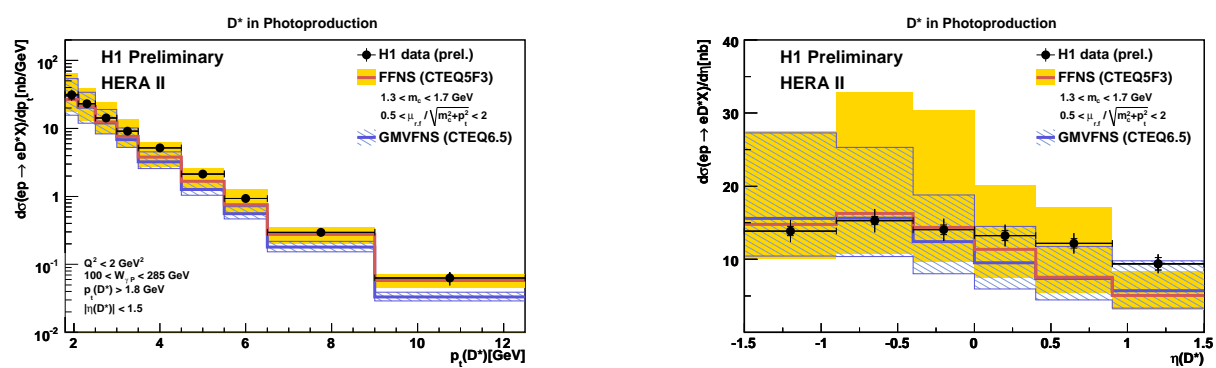

Figure 2: Differential cross sections as a function of $p_{t}\left(D^{*}\right)$ and $\eta\left(D^{*}\right)$. The measurements are compared to two NLO predictions calculated using FFNS and GMVFNS, respectively [3].

\section{Beauty in dijet photoproduction using electrons}

In this analysis done by the ZEUS collaboration the heavy quarks were tagged via their semileptonic decays into electrons [4]. A dataset corresponding to a luminosity of $120 \mathrm{pb}^{-1}$ with events containing at least 2 jets with $E_{T}^{\text {Jet 1(2) }}>7(6) \mathrm{GeV}$ and $\left|\eta^{\text {Jet1(2) }}\right|<2.5$ was selected. In order to separate the beauty and charm signal from the background, five discriminating variables sensitive to electron identification as well as to semileptonic decays were used. For the electron identification the specific energy loss, $d E / d x$, the fraction of energy deposited in the electromagnetic part of the calorimeter and $E / p$ were selected. Semileptonic decays were identified with the help of the angle $\Delta \phi$ between the missing transverse momentum, $p_{T}^{m i s s}$, which accounts for the nondetected neutrino, and the electron candidate, as well as the transverse momentum of the electron relative to the jet axis, $p_{t}^{r e l}$. The discriminating variables were combined in a likelihood fit in order to extract the fractions of events containing $b$ quarks and those containing $c$ quarks. Differential cross sections as a function of $p_{T}^{e}$ and $\eta^{e}$ are shown in Fig. 3. They are compared to the PYTHIA LO+PS Monte Carlo (MC) predictions [5] scaled according to the fit as well as to the NLO QCD predictions calculated with the FMNR programme. The predictions are in good agreement with the measurement. The charm cross sections were obtained in the same way [4].

\section{Beauty in dijet photoproduction using muons}

Another approach for heavy flavour lepton tags is the use of semileptonic muons in the final state. Two very similar analyses were performed by the H1 and ZEUS collaborations using datasets corresponding to a luminosity of $170 \mathrm{pb}^{-1}$ and $124 \mathrm{pb}^{-1}$, respectively [6, 7]. In both analyses, photoproduction events with at least 2 jets with $p_{T}^{\text {jet } 1(2)}>7(6) \mathrm{GeV}$ were selected. The muon candidates were reconstructed in the region $-0.55<\eta^{\mu}<1.1$ (H1) and $-1.6<\eta^{\mu}<1.3$ (ZEUS) and had to have a transverse momentum $p_{t}^{\mu}>2.5 \mathrm{GeV}$ (H1) and $1.5 \mathrm{GeV}$ (ZEUS). For the separation of the $b$ signal from charm and light flavour background events two discriminating variables were used: the impact parameter, $\delta$, is defined as the point of closest approach in $x-y$ of the track helix of the muon candidate with respect to the interaction point. It has a positive sign if the track crosses the axis of the associated jet within the jet hemisphere, otherwise 

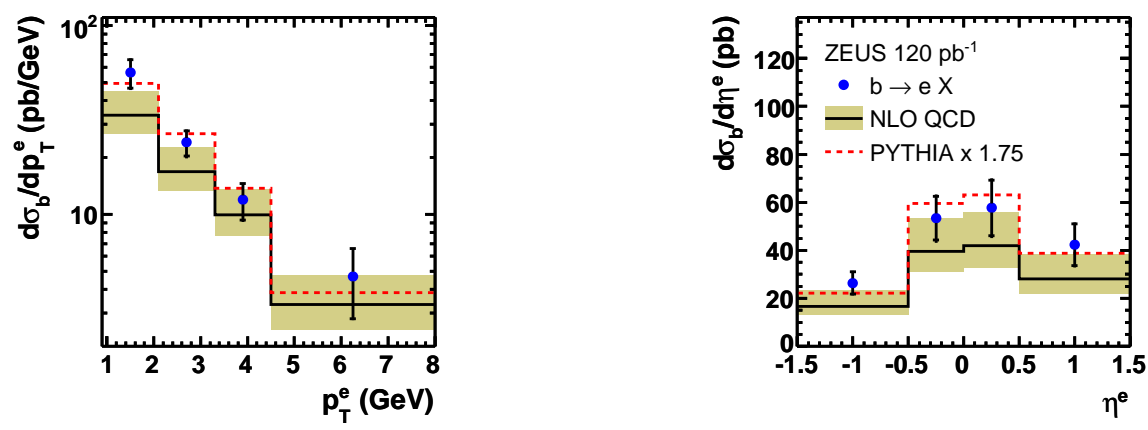

Figure 3: Differential cross sections as a function of $p_{T}^{e}$ and $\eta^{e}$. The measurements are compared to the scaled PYTHIA LO+PS MC as well as to the NLO FMNR predictions [4].

$\delta$ is negative. The second discriminating variable is $p_{t}^{r e l}$. The fraction of events containing $b$ quarks was determined from a simultaneous fit of the impact parameter and $p_{t}^{\text {rel }}$ distributions. Corresponding control plots for the ZEUS analysis are shown in Fig. 4. The data are compared to the PYTHIA LO+PS MC predictions. Good agreement between data and MC was observed. For the $\mathrm{H} 1$ analysis differential cross sections as a function of $p_{t}^{\mu}, \eta^{\mu}$, $p_{t}^{j e t 1}$ and the azimuthal angle between the two jets, $\delta \phi_{j e t s}$, were calculated (see Fig. 5). The measurements were compared to the PYTHIA LO+PS MC and to QCD NLO predictions calculated with the FMNR programme. Good agreement between data and NLO predictions is observed, while the PYTHIA MC also describes the shapes well. Furthermore the new measurements

\section{ZEUS}
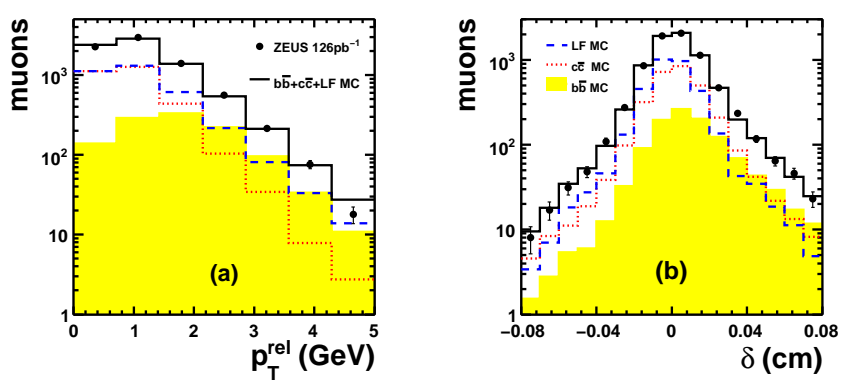

Figure 4: $p_{t}^{r e l}$ and impact parameter, $\delta$, control plots with the contributions from $b, c$ and light quarks being shown separately as denoted in the plots. [6].

were compared to a previous $\mathrm{H} 1$ result and found to be closer to the NLO predictions, while the previous measurement showed significant discrepancies at low $p_{t}$.

For the ZEUS analysis differential cross sections as a function of $p_{t}^{\mu}, \eta^{\mu}, p_{t}^{\mu-j}$ and $\eta^{\mu-j}$ were measured (see Fig. 6), here $\mu$-j means the jet containing the muon. The measurements were compared to the PYTHIA LO+PS MC and to QCD NLO predictions calculated using the FMNR programme. Good agreement with the NLO predictions as well as the scaled PYTHIA MC was observed. The differential cross section as a function of $\eta^{\mu}$ was in addition compared to results from a previous analysis, in which an external constraint on charm had to be applied. In the present analysis such a constraint was not needed anymore and the charm contribution was left as a free parameter in the fit. The results were found to be in very good agreement. 

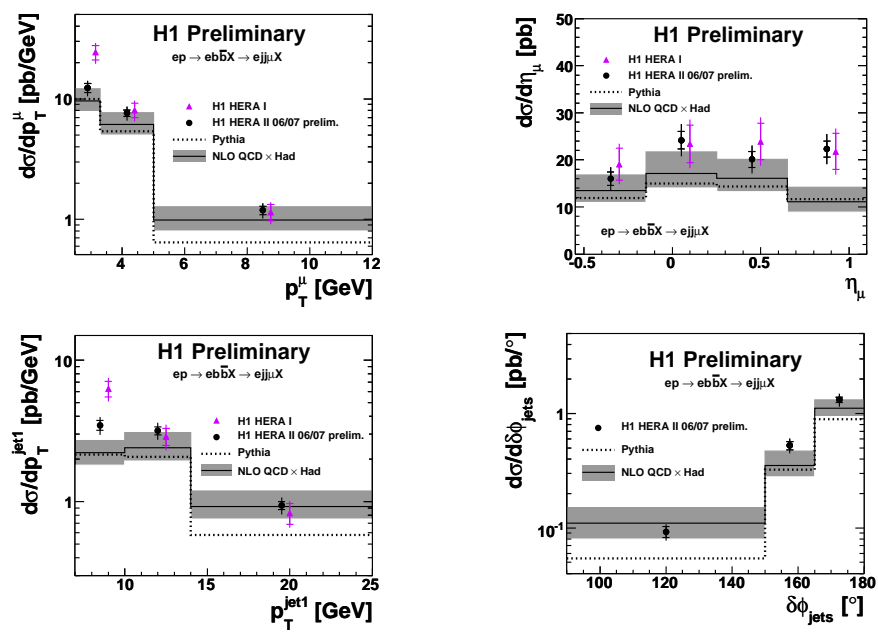

Figure 5: Differential cross sections as a function of $p_{t}^{\mu}$, for $p_{t}^{\mu}>2.5 \mathrm{GeV}, \eta^{\mu}, p_{t}^{j e t 1}$ and $\delta \phi_{j e t s}$. The measurements are compared to the PYTHIA LO+PS MC as well as the NLO FMNR predictions [7].

\section{ZEUS}
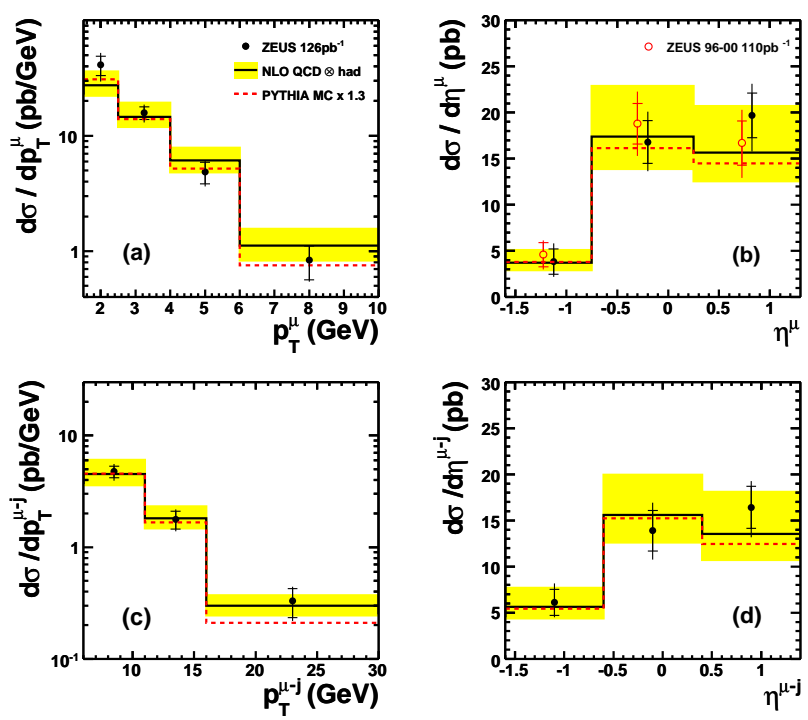

Figure 6: Differential cross sections as a function of $p_{t}^{\mu}$, for $p_{t}^{\mu}>1.5 \mathrm{GeV}, \eta^{\mu}, p_{t}^{\mu-j}$ and $\eta^{\mu-j}$. The measurements are compared to the scaled PYTHIA LO+PS MC as well as the NLO FMNR predictions [6].

\section{Beauty in dijet photoproduction using inclusive secondary vertexing}

In this analysis done by the ZEUS collaboration [8] the heavy quarks were tagged without imposing any requirements on the B hadron decay channels. Therefore the measurement could be kept fully inclusive, resulting in a large gain in statistics. A dataset corresponding to an integrated luminosity of $128 \mathrm{pb}^{-1}$ with photoproduction events containing at least 2 jets with $p_{T}^{\text {Jet } 1(2)}>7(6) \mathrm{GeV}$ was selected. For the separation of the beauty signal from charm and light flavours a secondary 
vertexing technique was used, exploiting the long lifetime of the B hadrons. Secondary vertices were fitted from well-reconstructed tracks associated to jets. Subsequently the 2-dimensional decay length, $d$, was calculated as the distance in $x-y$ between the secondary vertex and the beam spot, which was then projected onto the jet axis. In order to further improve the separation between signal and background the decay length was combined with the invariant mass, $m_{v t x}$, of the tracks fitted to the corresponding secondary vertex. In Fig. 7 the decay length significance, $S=d / \delta d$, is displayed for $2 \mathrm{GeV}<$ $m_{v t x}<7.5 \mathrm{GeV}$, showing that for large $m_{v t x}$ and high significances the asymmetry of the decay length significance for events containing $b$ quarks is large and an almost pure beauty sample can be obtained, allowing a direct comparison with the beauty $\mathrm{MC}$ prediction. The beauty-enriched region with $m_{v t x}>2 \mathrm{GeV}$ and $S>8$ was found to be welldescribed by this Monte Carlo. For the extraction of the beauty contribution in the data, the negative part of the decay length significance distribution was mirrored and subtracted from the positive part, in order to minimize the light flavour contribution and to cancel out potential sys-

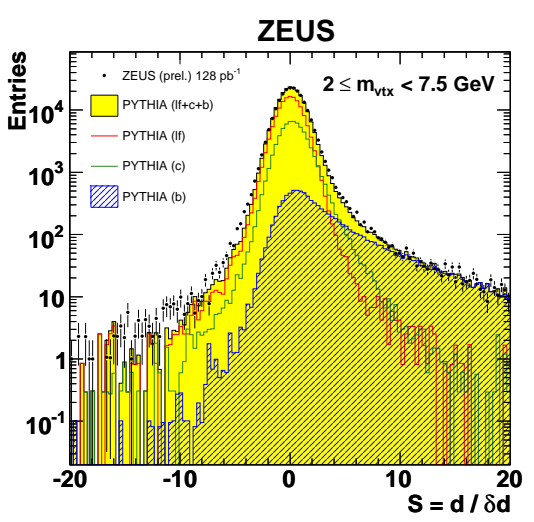

Figure 7: Decay length significance, $S$, for $2 \mathrm{GeV}<m_{v t x}<7.5 \mathrm{GeV}[8]$ tematic effects. Subsequently, the mirrored distribution, $S^{+}-S^{-}$, was split into three bins of $m_{v t x}$ with the third bin being highly beauty-enriched, while the other two were dominated by charm. The beauty contribution was then determined from a $\chi^{2}$-fit of the beauty, charm and light flavour MC distributions to the data, simultaneously performed in all three $m_{v t x}$ bins. Differential cross sections as a function of $p_{T}^{\text {Jet }}$ and $\eta^{\text {Jet }}$ were measured and compared to the scaled PYTHIA MC predictions as well as to QCD NLO predictions calculated using the FMNR programme (see Fig. 8). Good agreement between the measurements and both predictions was observed.
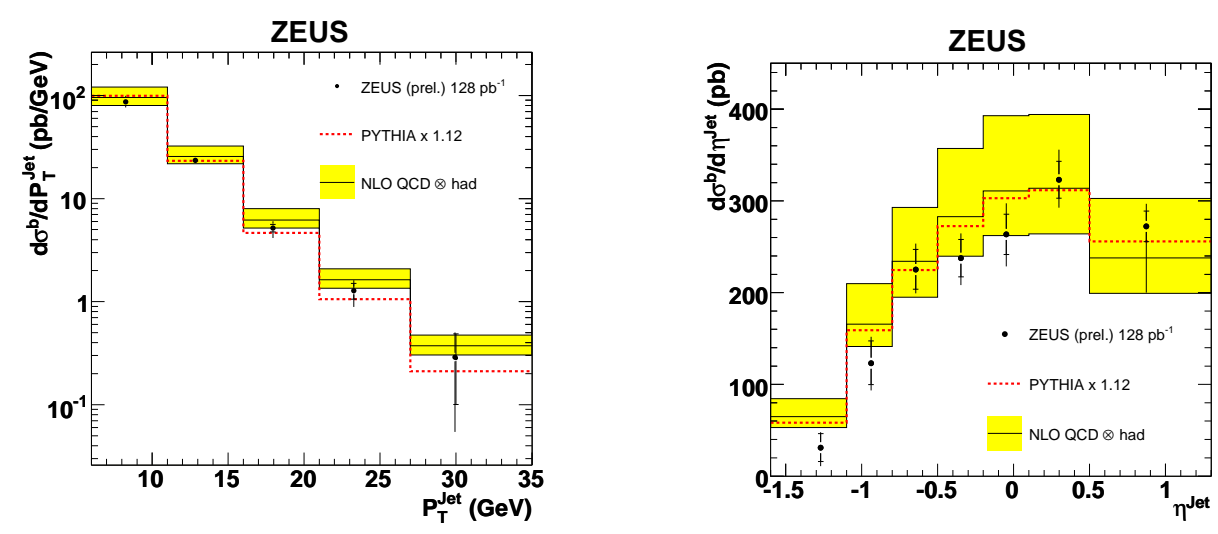

Figure 8: Differential cross sections as a function of $P_{T}^{\text {Jet }}$ and $\eta^{\text {Jet }}[8]$.

The differential cross sections as a function of $\eta^{\text {Jet }}$ were additionally compared to the results of a previous analysis [9]. The measurements were found to be in good agreement, with the new result substantially increasing the precision of the measurement. 


\section{Summary}

A selection of the latest results on heavy flavour photoproduction at HERA were presented. New methods for beauty and charm tagging were used and improved, among them various lifetime tagging techniques. The first ZEUS analysis on beauty production from inclusive secondary vertexing was presented, which in contrast to the exclusive analyses has the advantage of not being restricted to a specific B hadron decay channel. It can be concluded that the various $\mathrm{H} 1$ and ZEUS measurements using different experimental techniques are in very good agreement, as is displayed in the summary plot in Fig. 9, with the most recent measurements being denoted by open crosses, downward-pointing triangles and filled stars. The measured cross sections as a function of $p_{T}$ of the $b$

HERA

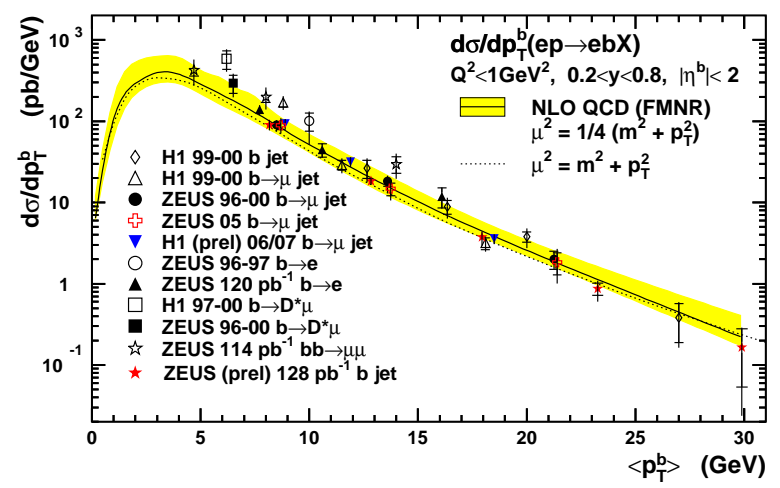

Figure 9: cross sections for beauty production as a function of $p_{T}^{b}$ from various $\mathrm{H} 1$ and ZEUS measurements.

quark are shown for several independent H1 and ZEUS analyses and compared to the NLO QCD prediction calculated using the FMNR programme. A general agreement with the NLO prediction is observed, giving a consistent picture of $b$ quark photoproduction over a wide range of $p_{T}^{b}$.

\section{References}

[1] S. Frixione et al., Heavy quark correlations in photon-hadron collisions, Nucl. Phys. B 412, (1994) 225 [arXiv: hep-ph/9306337v1]

[2] B.A. Khniel et al., Collinear Subtractions in Hadroproduction of Heavy Quarks, Phys. Rev. C 41, (2005) 199 [arXiv: hep-ph/0502194v1]

[3] H1 Collaboration, Measurement of $D^{*}$ Meson Production in Photoproduction, H1prelim-08-073

[4] ZEUS Collaboration, S. Chekanov et al., Beauty photoproduction using decays into electrons at HERA, Phys. Rev. D 78 (2008) 072001 [arXiv : 0805.4390]

[5] T. Sjöstrand et al., Pythia 6.2 - Physics and Manual, 2002, http://www.thep.lu.se/ torbjorn/pythia/pythia6206.pdf

[6] ZEUS Collaboration, S. Chekanov et al., Measurement of beauty photoproduction using decays into muons in dijet events at HERA, JHEP 04 (2009) 133 [arXiv: $0901.2226 \mathrm{v} 2$ ]

[7] H1 Collaboration, A Measurement of Beauty Photoproduction Through Decays to Muons and Jets at HERA-II, H1 prelim-08-071

[8] ZEUS Collaboration, Measurement of beauty photoproduction from inclusive secondary vertexing at HERA II, ZEUS-prel-09-005

[9] ZEUS Collaboration, S. Chekanov et al., Beauty photoproduction measured using decays into muons in dijet events in ep collisions at $\sqrt{s}=318 \mathrm{GeV}$, Phys. Rev. D 70 (2004) 012008 [arXiv: hep-ex/0312057v2] 